\title{
diffusion-îuridarrentals
}

The Open-Access Journal for the Basic Principles of Diffusion Theory, Experiment and Application

\section{Optimal $k$-space Sampling for Single Point Imaging of Transient Systems}

\author{
Prodromos Parasoglou ${ }^{1}$, Andrew J. Sederman ${ }^{1}$, John Rasburn ${ }^{2}$ and Hugh Powell ${ }^{2}$ and \\ Michael L. Johns ${ }^{1}$ \\ ${ }^{I}$ Department of Chemical Engineering, University of Cambridge, CB2 3RA, UK \\ ${ }^{2}$ Nestlé Product Technology Centre, York, UK
}

Corresponding author: Dr Michael L. Johns, Dept. of Chemical Engineering, University of Cambridge, New Museums Site, Pembroke Street, Cambridge, CB2 3RA, E-Mail:

mlj21@cam.ac.uk

(received 4 July 2008, accepted 18 January 2009)

\begin{abstract}
A modification of the Single Point Imaging (SPI) is presented. The novel approach aims at increasing the sensitivity of the method and hence the resulting Signal-to-Noise ratio $(S N R)$ for a given total time interval. With prior knowledge of the shape of the object under study, a selective sparse $\boldsymbol{k}$-space sampling can then be used to follow dynamic phenomena of transient systems, in this case the absorption of moisture by a cereal-based wafer material. Further improvement in the image quality is achieved when the un-sampled $\boldsymbol{k}$-space points are replaced by those of the initial dry or the final wet sample acquired at the beginning and the end of the acquisition respectively when there are no acquisition time limitations.
\end{abstract}

\section{Keywords}

$\boldsymbol{k}$-space, SPI, selective sampling, rapid imaging

\section{Method Development}

\subsection{SNR Optimisation for a Known Geometry}

SPI [1] is a transient magnetisation imaging technique [2].Consequently each point in $\boldsymbol{k}$ space is sampled with a different amount of magnetisation. The SNR ratio for an image is predominantly determined by points acquired at low $\boldsymbol{k}$ values (corresponding to lower spatial frequencies [3]) .Hence more optimal use of the available magnetisation, before it reaches its equilibrium value, is achieved via sampling trajectories that are initiated at the $\boldsymbol{k}$-space origin. In the work presented here, an alternative way of sampling $\boldsymbol{k}$-space is proposed that is suitable when prior knowledge of the system shape exists. Instead of a general geometric $\boldsymbol{k}$-space trajectory [4], a trajectory that maximises the SNR is implemented. The relatively simple principle employed is that $\boldsymbol{k}$-space points with the highest probable modulus signal intensity are acquired with the highest value of magnetisation [5]. 


\subsection{Selective Sparse $\boldsymbol{k}$-space Sampling}

SPI compared to frequency encode techniques has, in general, longer acquisition times. To follow dynamic changes in transient systems an attempt is made to accelerate acquisition. Geometric knowledge of the object of interest enables via inverse Fourier Transform (e.g Figure 1) a rank order of importance to be assigned to the $\boldsymbol{k}$-space points based on their modulus signal intensity. Hence, by sorting them one can decide to omit a significant amount of points that will contribute least in the final image. Finally, the $\boldsymbol{k}$-space points with the least information can either be zero-filled prior to Fourier transform, or can be replaced by $\boldsymbol{k}$-space points acquired for the same sample at the beginning or the end of acquisition consisting of a series of images tracking a transient phenomenon [5].

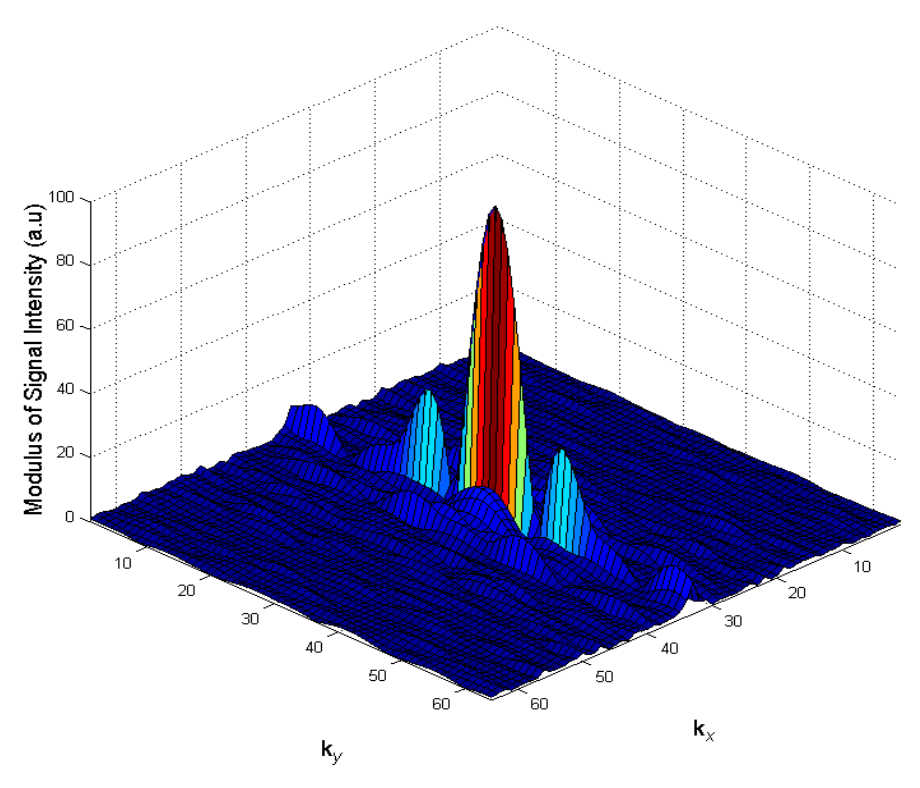

Fig. 1: Signal from the cross-sectional image shown on Fig 2. Lower spatial frequencies in general have a higher modulus. However, there are points further away from the origin with a significant amount of information.

\section{Experimental Results}

All experiments were conducted using a Bruker AV400 spectrometer equipped with a micro-imaging r.f. coil of $25 \mathrm{~mm}$ internal diameter. The samples were placed in a $17 \mathrm{~mm}$ internal diameter NMR tube featuring a glass sample holder and connected to a humidified air flow which ranged from 0 to $250 \mathrm{ml} \mathrm{min}^{-1}$. The sample dimensions are shown in Figure 2. Images of the wafer were acquired whilst humid air was blown over it. Imaging of the wafer samples is very challenging as the porosity of the samples is typically $80-90 \mathrm{vol} \%$ and the absorbed moisture is typically only $2-7 \mathrm{wt} \%$ of the solid content. $T_{2} *$ for water, is typically of the order of $50 \mu \mathrm{s}$. Imaging of this system with conventional frequency encode methods is not possible, hence the use of single point techniques is necessitated. The moisture signal is also, however, characterised by a relatively long longitudinal relaxation time, $T_{1}(\sim 1 \mathrm{~s})$. A sample result is presented in Figure 3. In the above system a full acquisition of a $64 \times 64 \boldsymbol{k}$ space matrix with 16 signal averages requires 40 minutes. Acquisition time in the sparse imaging reduces proportionally to the percentage of acquired $\boldsymbol{k}$-space.

\section{Conclusion}

We have demonstrated that with prior knowledge of a general sample of interest's shape, a sparse $\boldsymbol{k}$-space sampling strategy in an SPI experiment can be optimised with respect to $S N R$ for a given acquisition time interval. Furthermore, a full $\boldsymbol{k}$-space acquisition at the beginning and the end of the dynamic process can give information of the lower spatial frequencies that 
can be used to fill in the un-sampled points and give improved quality images.
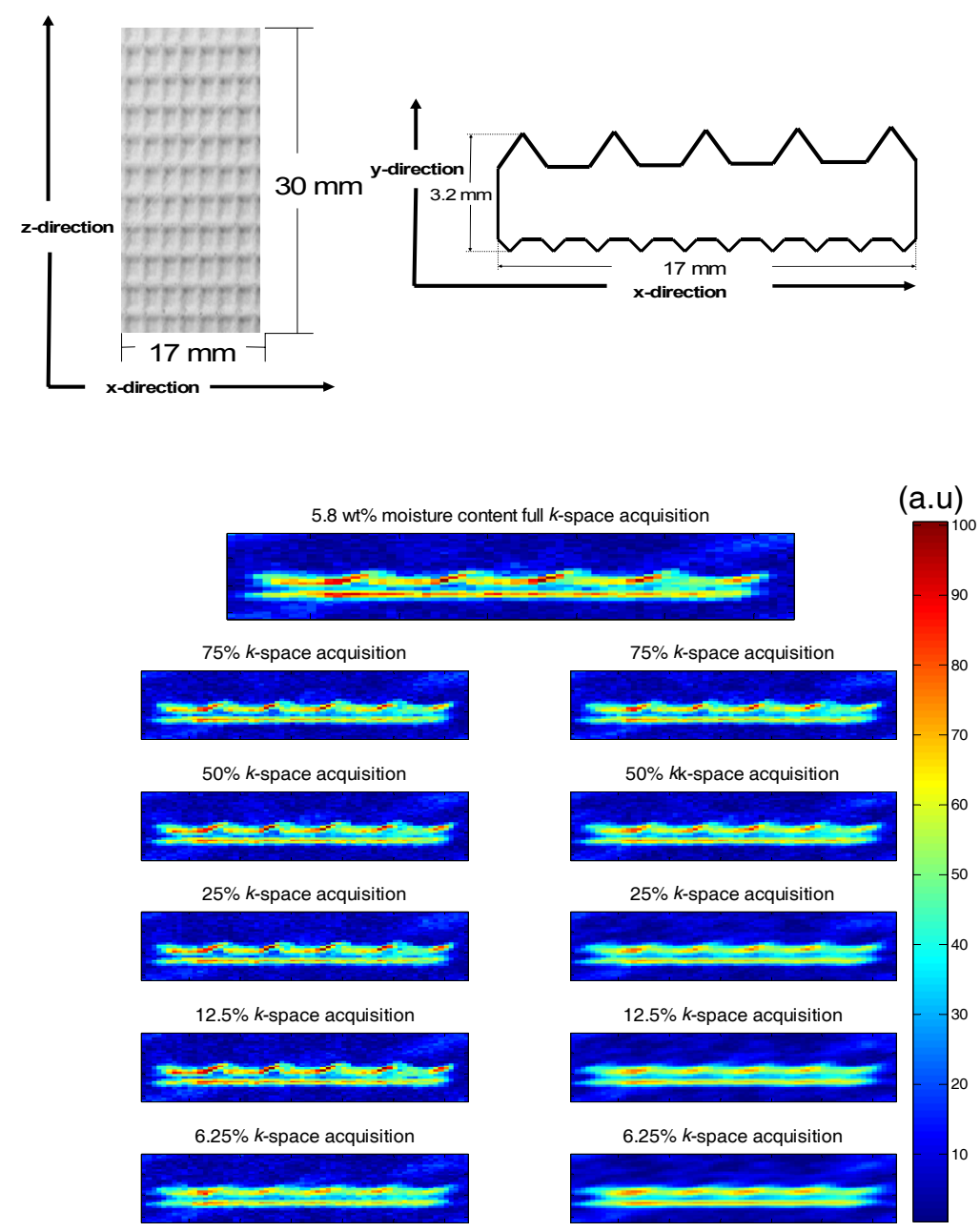

\section{References}

[1] S. Emid, and J.H.N. Creyghton, High-resolution imaging in solids. Physica B \& C 128 (1985) 81-83.

[2] I.V. Mastikhin, B.J. Balcom, P.J. Prado, and C.B. Kennedy, SPRITE MRI with prepared magnetization and centric k-space sampling. Journal of Magnetic Resonance 136 (1999) 159-168.

[3] J.C. Russ, Image Processing Handbook, Fourth Edition, CRC Press, Inc., 2002.

[4] A.A. Khrapitchev, B. Newling, and B.J. Balcom, Sectoral sampling in centric-scan SPRITE magnetic resonance imaging. Journal of Magnetic Resonance 178 (2006) 288-296.

[5] P. Parasoglou, A.J. Sederman, J. Rasburn, H. Powell, and M.L. Johns, Optimal k -space Sampling for Single Point Imaging of Transient Systems. Journal of Magnetic Resonance (2008) doi:10.1016/j.jmr.2008.06.005. 